\title{
New architecture for an Ultra Broadband Home Area Network with spread connectivity and autonomic functions.
}

\author{
Pierre Jaffré ${ }^{1}$, Romain Insler' ${ }^{1}$, Vasilis Freiderikos ${ }^{1}$, Sylvain $_{\text {Meyer }}{ }^{1}$ and \\ Martial Bellec ${ }^{2}$ \\ 'France Telecom Research \& Development, 2 Av. Pierre Marzin 22307 Lannion, France \\ ${ }^{2}$ France Telecom Research \& Development, 4 rue du Clos Courtel 35512 Cesson Sevigne, \\ France \\ Email: name.surname@orange-ftgroup.com
}

\begin{abstract}
Operating Local Area Networks, in the home of their customers, has recently become a new business for Network Operators. On the other hand, Gigabit optical technologies are introduced in the operators' access network and new digital terminals appear in the customer premises, such as HDTV videos, PCs and home servers, requiring more and more a high speed data transfer inside the apartment. These evolutions will appeal for the emergence of an Ultra Broadband Home Area Network (UBB-HAN) with advanced connectivity capabilities that will combine many technologies. We introduce a new architecture for this future UBB-HAN which includes some foreseeable autonomic functions to cope with the management and the increased complexity of such a heterogeneous HAN.
\end{abstract}

\section{CONTEXT}

\subsection{The Home Area Network is a new business for Network Operators}

In 2004, France Telecom started the commercialization of a Home Gateway named "Live Box". After three years, about 5 Millions of these Customer Premise Equipments (CPE) are in operation in European countries. Services such as TV programs or Video on Demand over xDSL [1] are already a commercial success. But beside the quadruple play services that this box provides, customers use it as a Home Networking tool.

\subsection{Emergence of Gigabit optical technologies in the Access Network}

In 2006, France Telecom introduced GPON systems in its access network within the framework of a "Very High Speed" program for a

Please use the following format when citing this chapter:

Jaffré. P., Insler, R., Friederikos, V., Meyer, S., Bellec. S.. 2007, in IFIP International Federation for Information Processing, Volume 256. Ilome Networking, Al Agha. K., Carcelle. X., Pujolle. G., (Boston: Springer). pp. 151-162. 
FTTH offer [2]. FTTH is today widely deployed in countries like Japan and Korea, while in Europe and in the US several companies are developing detailed commercial plans for a mass deployment. It is thus possible to envision in the near future a scenario in which a large amount of users will be offered a very high performance connection up to the "main door" of their apartment. Several hundreds of Mbps are reasonably reachable in the coming future, backed up by emerging standards such as GPON 2.4 G.984 [3]. A Dynamic Band Assignment mechanism allows the system to dedicate several hundreds of $\mathrm{Mbps}$ to a single user for a short time period, when bandwidth is available.

\subsection{Requirement of Gigabit Data Rates in the Home Area Network}

Digital Mass storage devices gain more success to the home every day. These devices, whose standardization is ongoing by e.g. within DLNA [4] or HGI [5] fora, offer not only demodulation of digital broadcast programs, access to remote operator services but also high connectivity to end devices such as TVs, home cinema or PCs. To enable the use of these devices, the trend is that it shall be possible to use them everywhere at home with high data rate connectivity to transfer content either from remote servers or between end devices sparsely distributed everywhere at home. Moreover, end user devices are fitted with high speed interfaces to easily transfer all types of multimedia supports. In the coming future these trends will certainly make the UBB-HAN a convergence arena where these devices and services will have to interoperate at home and in continuity with the operator's network. A well connected home will need an internal network operating at data rates around $1 \mathrm{Gbit} / \mathrm{s}$ by 2010 [6].

\subsection{Necessity for a new architecture for Ultra Broadband Home Area Networks}

Until now, with DSL technology in the access network, customers connected their box to a telephone set, locating the Access Point of their Home Network in the central part of their living space. With the optical technology at the access, a very high performance connection will be available in front of the Optical Network Termination (ONT) that is located near the "main door" of their apartment. It is anticipated that some of the gateway functions enabling connection to the service platforms through the network will be integrated to the ONT. On the other hand, a Gigabit coverage of the living space will require more than one AP, with probably several technologies involved. To guarantee the coverage, we anticipate the necessity to hybridize many connectivity technologies that can be divided in 3 types:

- Wireless, like radio or Free Space Optics,

- Wired, but without installation of a new wire, like Power Line Communication or over phone lines, like HomePNA or MoCA... 
- Wired, with installation of a copper or optical physical media required

From the operator's point of view, it is essential to guarantee the Quality of Service of his own services transported over this complex Home Network. In particular, the quality evaluation of links using different technologies has to be homogeneous. Congestions have to be managed, with eventually reconfiguration of the network. Finally, it is essential for an operator to protect his own flows from local flows that can require resources on the same Home Network.

\section{New architecture for an Ultra Broadband Home Area Network}

\subsection{The concepts of bridge and backbone in the UBB-HAN}

A UBB-HAN requires new functionalities to improve its coverage and performance. A potential solution would implicate the distribution of numerous access points supporting different connectivity technologies. We present hereafter a new architecture answering these requirements (figure 1). Its main components are:

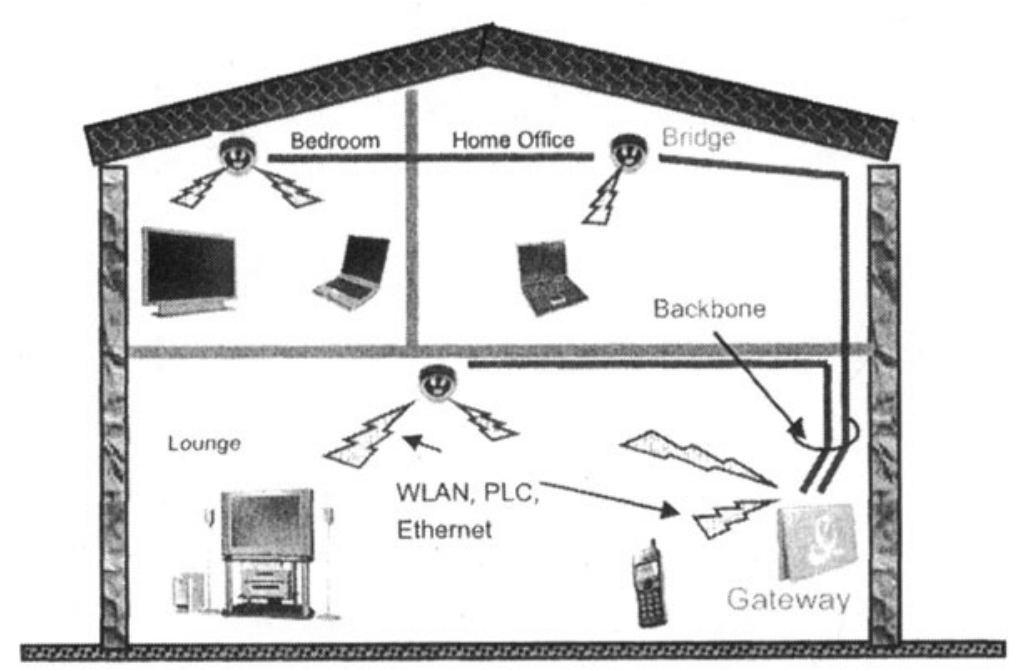

Figure 1: Outline of the Ultra Broadband - Home Area Network Architecture

- Home Gateway: interface between the access and the home network. The gateway may be just an interface without any other functions or even a more complex device with advanced functionalities. 
- Bridge: node of the home network. This new device spreads the connectivity and improves home network coverage and performance. The bridge answers specific requirements newly identified within the UBB-HAN (availability of very high bandwidth, differentiated QoS, alternative route in case of congestion or link failure)

- Backbone: a very high data rate core constituted by the various bridges and the gateway. It may involve any high performance connectivity technology. The backbone network must provide a back-up solution in case of link congestion or failure.

\subsection{Innovative techniques for the UBB-HAN architecture}

The Ultra Broadband - Home Area Network (UBB-HAN) can be presented as a set of segments made up each one by a technology of connectivity (plastic or silica optical fibre, radio over fibre, free space optics, UWB, WiFi, PLT, Ethernet cabling...). Each segment carries several terminals, and the various segments are inter-connected by the Home Gateway and the bridges. This architecture represents a new situation where several paths and connectivity modes can be chosen to interconnect the various devices of the LAN. (example of figure 2 where a dual-mode terminal WiFi/UWB is connected to another PLT terminal either through the Home Gateway or through the bridge).

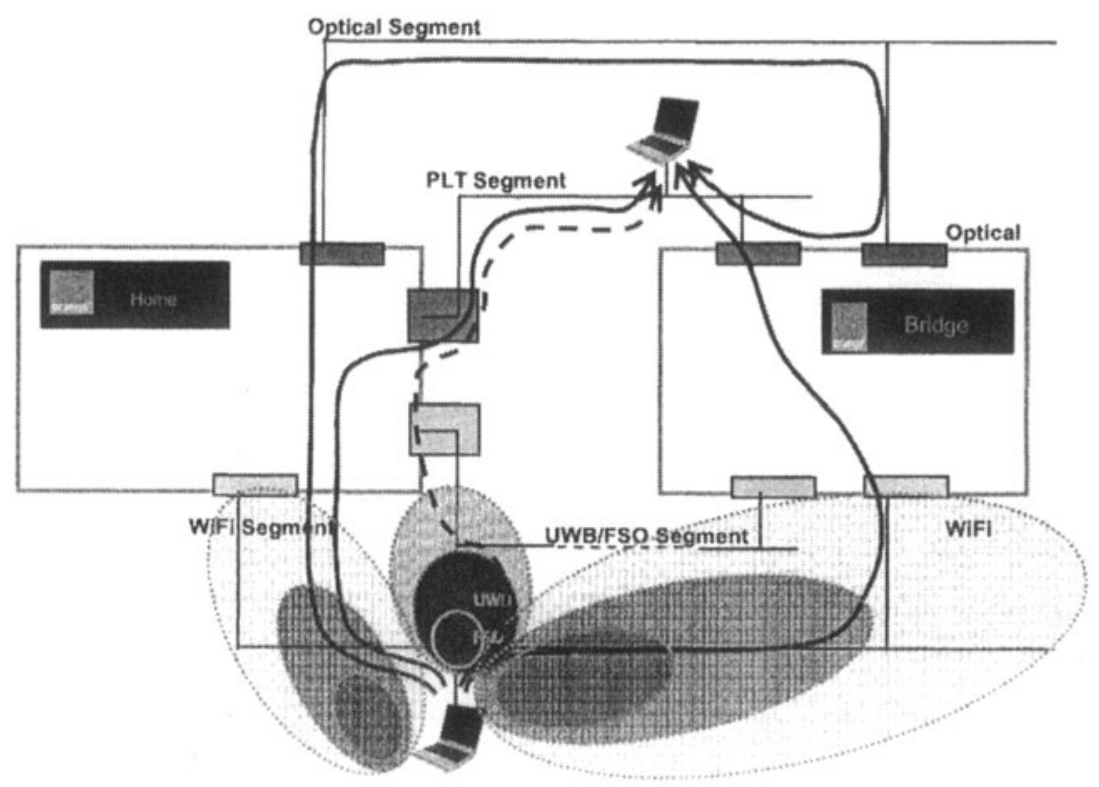

Figure 2: Illustration of path \& connectivity selection functions in the UBB-HAN 
According to whether this function of path selection is concentrated on the level of the Home Gateway or distributed between the Home Gateway and the bridges, one makes the distinction between centralized architecture (around the Home Gateway) and distributed architecture.

In the centralized approach, the network can be organized either in a tree or star structure around the Home Gateway (HGW) which will act as the main home manager, whereas the bridges act as relays. The role of the bridges is twofold, firstly, to extend the coverage to the whole home and secondly to update the HGW with the information on link status. Therefore, the network topology management is performed in a centralized way by the HGW: the forwarding function is coordinated by the HGW.

As illustrated in figure 3, the traffic is handled by disseminating the routing policies from the HGW to the network elements which will enforce them. Indeed, the HGW makes a centralized decision of a path, and then it establishes the path by informing all the bridges of the relevant path information. Note that in some specific scenarios the whole traffic may go through the $\mathrm{HGW}$ to perform specific processing (authentication,...). As a matter of fact, this approach should be simpler than the distributed one, but it could raise point of failure and bottleneck issues inside the HGW.

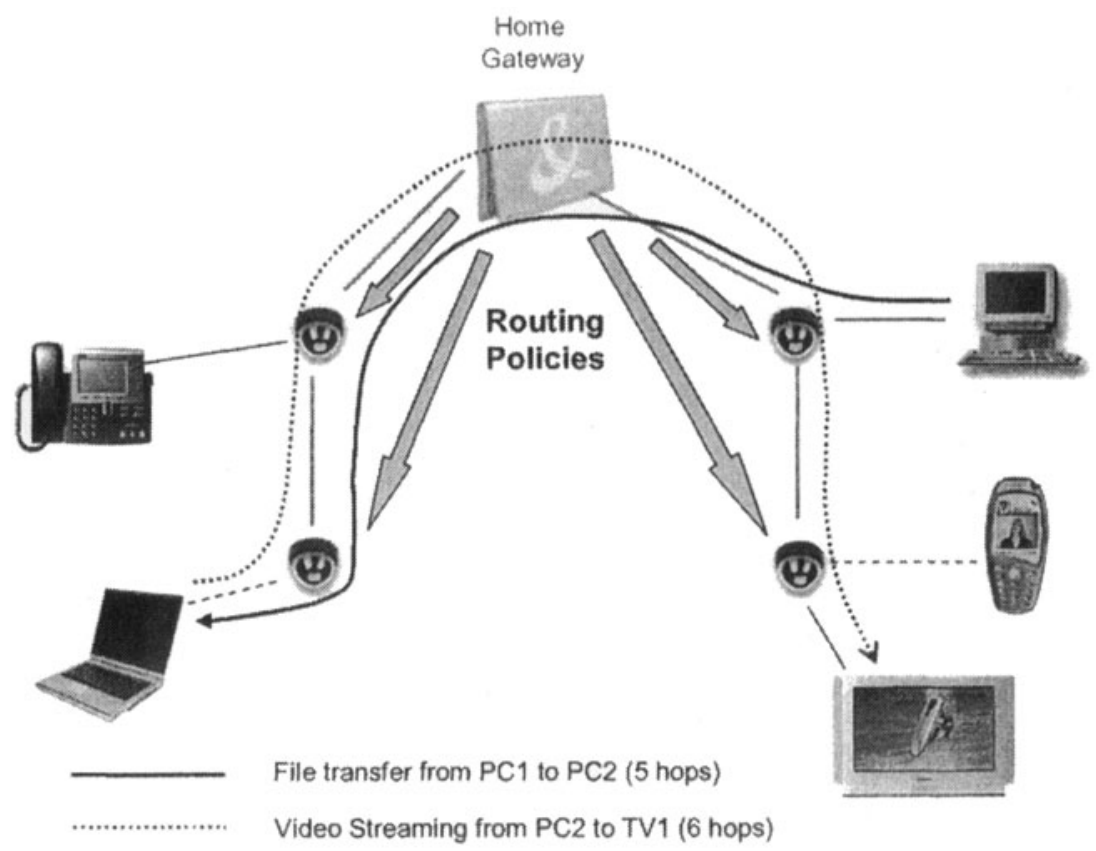

Figure 3: Centralized approach in the UBB-HAN 
In contrast with the centralized approach, in the distributed approach, the topology management (path selection and establishment decisions) is performed in a distributed manner between the bridges and the HGW in using mesh network principles. Indeed, the HGW and the bridges are interconnected without any predefined constraint forming therefore a mesh network. This approach implies that either proactive or reactive ad hoc forwarding protocols can be applied at each bridge for path computation and establishment.

For the deployment matter, the distributed model seems to be more upgradeable and more flexible. However, the distributed character of the solution bringing more reliability and scalability by avoiding the gatewaycentric routing effects, generate on the other hand some potential issues related in particular to the QoS insurance like resource reservation, scheduling and routing decision that requires nevertheless more efficient policies.

The Figure 4 illustrates how the forwarding of flows is performed in the case of fully distributed approach.

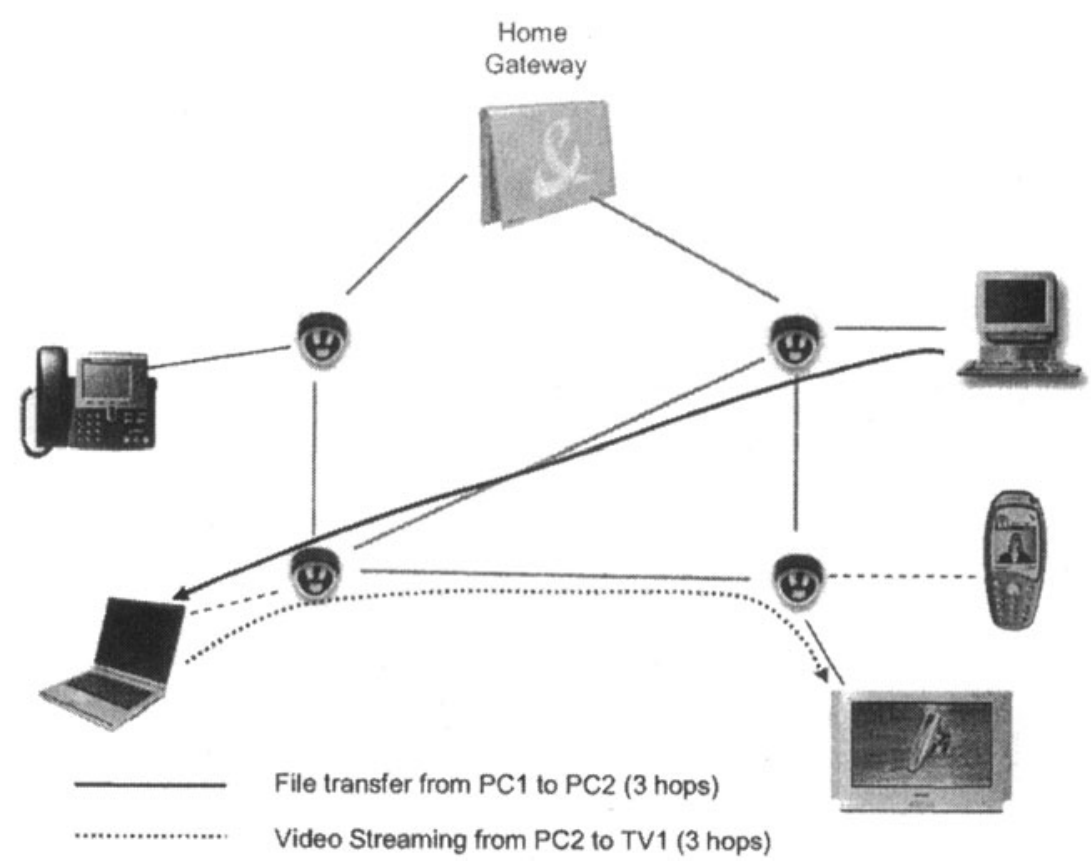

Figure 4: Distributed approach in the UBB-HAN

On the example of figure 4, one sees that the implementation of a mesh structure makes it possible to optimize the forwarding of flows regarding a criterion of a number of hops between source and destination. Some other metrics appear relevant for the choice of the path used for a given 

and autonomic functions.

flow : for example, one may choose the path presenting the best signal to noise ratio, or the path ensuring the best distribution of the traffic, with respect to the QoS aims of the system (packets error rate, latency, jitter).

Thus, the UBB-HAN seems to be a mesh structure mixing technologies, from where the idea to transpose radio mesh techniques to the context of a network based on heterogeneous technologies, cascading wired and wireless media.

Another innovating feature is related to the definition of a convergence layer making possible the cohabitation of very heterogeneous technologies of the UBB-HAN, in the context of the work initialized by authorities of standardization and various organizations of research (examples of the forum IEEE 802.21 [7] and of the Berkeley Wireless Research Center [8]). According to the context, this layer of convergence can actually address very different concepts, from the continuity between networks (in the IEEE 802.21) up to the interactivity between the services. In our case, associated to the function of path selection (and connectivity selection), it appears in a first step like a function ensuring the consistency of information collected at the level of the MAC layers of various technologies, or function of InterMAC co-operation. In a second step, it will be able to take into account the mechanisms ensuring the continuity of the UBB-HAN with the access.

By its highly interactive character, the UBB-HAN has a sensitivity particular to the concerns of configuration, safety, maintenance and management of the Quality of Service. In order to face up to them, the prospects for transposition to the UBB-HAN of the autonomic techniques imagined for the large networks can be taken into consideration. These techniques are indeed suitable to provide the networks with the capacity to react in an autonomous way to a situation of new configuration (example the customer modifies its installation), with a problem of safety, a local degradation of the conditions of transmission, or with an evolution of the distribution of the traffic, and that without external intervention of the customer or the information system of the operator, which is particularly critical in the situation of complexity of the UBB-HAN. This topic is addressed in the section 3 .

\section{Autonomic functions}

\subsection{Autonomic technologies}

Autonomic technologies represent a recent and ongoing effort to cope with the ever increasing complexity and heterogeneity of IT and communication systems. It's all about systems that are particularly designed in order to be able to manage themselves without any human 
intervention. They introduce a plethora of so-called self-* properties, such as self-healing, self-configuration, self-protection, self-optimization [9].

In the world of communication networks, an autonomic architecture should consist of network elements that are able to sense their environment, collect information about its operational state, analyze these data, make decisions and plan future configurations and finally reconfigure themselves. In a more synergic scenario, these network elements should be capable of communicating with each other, and sharing their resources in order to control the system's global behavior.

\subsection{Knowledge plane and 4-plane architecture.}

In the "Authone" Project [10] a 4-plane architecture is proposed in order to "inject" the intelligence of an autonomic system in the home area network. We will use a similar model to describe the autonomic behavior and expectations of our UBB-HAN. This architecture involves the following planes:

- The data plane which includes all the functions, operations and services related to layers $1-4$ of traditional networks.

- The knowledge plane which is responsible to collect any significant information for the state of the network produced by the data plane, in order to ensure optimal resource management, QoS, traceability and mobility.

- The governance plane which processes the data of the knowledge plane, in order to make decisions concerning the reconfiguration of the system.

- The control plane, which contains reactive agents that act on the controlled elements to achieve the goals imposed by the governance plane.

\subsection{Autonomic functions for a UBB-LAN.}

Back to our architectural model, one can imagine the data plane like the source of operational information for the "gateway" and the various "bridges" (and possibly of certain terminals) which feeds the knowledge plane. This one can be thought of as a database system which provides the governance plane with an almost complete image of these network elements' state. The governance plane has the role of the system's "brain"; it could be an intelligent agent which exploits the information of the knowledge plane and based on decision algorithms plans, when it is necessary, the reconfiguration of the system. These algorithms can be as well simplistic (preset rules in collaboration with a policy-based management system), as well as more sophisticated, such as self-learning systems that use their experience in order to improve their performance (e.g.: neural networks). Last in the chain of interaction, is the control 
plane, a kind of "actuator" agent, which applies the new configuration to the concerned network elements.

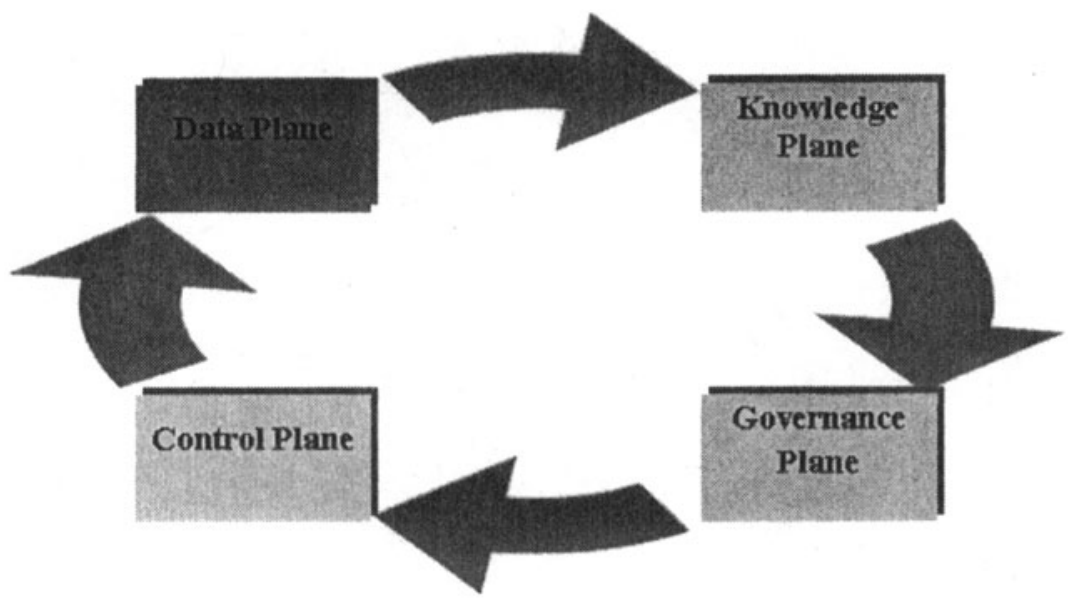

Figure 5: Plane interaction model

Given the home network environment such as it is described earlier (Par.2), we can consider various application scenarios for the autonomic technologies, all aiming at making the HAN intelligent and self-managed. The following figure (fig.6) represents the UBB-HAN as a mesh network structure, but this time extended by a knowledge and a control plane on each element of the residential backbone. Each node of the backbone has a situated view of the network, which means that it is conscious of the current state of network links and nodes in its neighborhood (ex.: 1 hop). This information can be shared on request with the knowledge plane of neighbor nodes, so that they can make decisions aligned with a global network configuration goal. The area covered by the situated view is a very important factor for the operation of this architecture. A large area produces more information to be exploited by the knowledge plane but also a more important "secondary" traffic dedicated to network management. An acceptable compromise must be chosen.

Combined procedure of path and connectivity selection: Traditional approaches for the resolution of this type of problems, often similar to routing algorithms, are likely to prove insufficient. A group of autonomous and interactive agents, able to feel the state of the links and other network elements and build a distributed knowledge plane, could possibly offer more adequate solutions. Moreover, a multi-agent system which functions independently of the network, can act in a proactive way in order to improve the performance of the transition in terms of time. In this case, the knowledge plane will comprise information such as link 
state, packet delay and transmission time between different nodes, available resources and quality of radio signals (SNR).

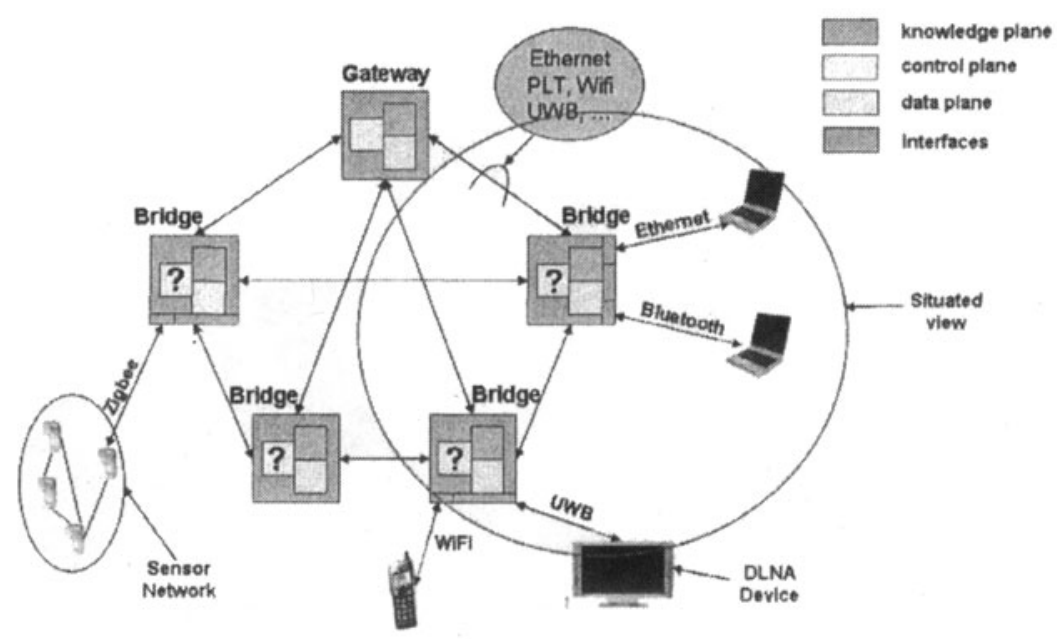

Figure 6: UBB HAN with Autonomic Functionalities.

UBB-LAN maintenance: In the same spirit as above, a distributed operational database could assist with the maintenance and the automated troubleshooting of the residential network. It is an essential functionality for the operator and his centralized management platforms which would have a heavy or even impossible mission to accomplish in a network of tens of million gateways and bridges. However, even we choose this distributed management structure for the knowledge plane, we can always keep in the gateway a summary of the different knowledge planes located on the bridges, for further processing by the operator's information systems.

Security: Security in a residential network imposes a minimum of feedback and handling by the end user, especially when it concerns authentication aspects. Thus, one could easily say that there is not much space for the deployment of autonomic technologies. However, they can be used to face other threats of security such as intrusions or "Denial of Service" attacks. For example, a system of intelligent agents in collaboration with a knowledge plane, able to analyze the traffic in any moment and discover abnormal flows, could answer these situations and ensure a transparent experience for the home user.

Fault diagnosis and troubleshooting: Automatic fault diagnosis and troubleshooting will be critical for the operation of an autonomic UBBHAN. L.Qiu and al. presented a simulation-based fault detection system for wireless mesh networks [11]. Inspired by their approach, we can consider a diagnostic system adapted to the autonomic principles. This 
system would be composed of two distinct software modules. An agent that will run on every node of the residential backbone, gathering information about various protocol layers and a manager that will be fed with the agent's data in order to analyze them and act in a suitable way. With respect to our main UBB-HAN architecture, we can choose between a manager that runs on a single node (centralized architecture), or in a set of nodes (distributed architecture). In a first step, agents gather and exchange their situated view of the network's operation. Then, the manager, using the experience of a knowledge plane properly built to describe the operational points of our network, decides if the observed result is normal for the given configuration and traffic model. A fault is detected when the expected performance is far from the observed performance. Root cause analysis is done by finding the set of faults which minimizes this difference. It remains to us to specify the exact mechanism of data analysis. A neural network well-trained in various operation conditions could produce reliable results.

Quality of Service Management (QoS): The wide diversity of coexisting services in the UBB-HAN forces us to invent new methods to protect flows coming from the operator's specific services with respect to flows generated locally in the residence. Moreover, it is important that we propose new congestion avoidance mechanisms to manage efficiently and automatically the various considered flows among devices with different characteristics and processing capacities:

- WAN $\rightarrow$ LAN (VoD, Broadcast TV, VoIP, Internet,...)

- $\quad$ LAN $\rightarrow$ WAN (Zapping for Broadcast TV, VoIP,...)

- $\quad$ LAN $\rightarrow$ LAN (File transfer PC to PC,...)

Lastly, one should conceive a new admission control and flow classification system adapted to the needs of a UBB-HAN. An autonomic system could assist with keeping the QoS contract in such a dynamic environment. Based on network state information, provided by the knowledge plane, it could improve the reaction performance of existing control algorithms, in cases such as bridge congestion or broken radio channel.

\section{Conclusions}

The penetration of bandwidth hungry services inside the home and the arrival of very high performance optic connection up to the "main door" will push the deployment of Ultra Broadband Home Networks. For a good coverage, these networks will combine many connectivity technologies, with Access Points spread over the home. This deployment will call for a more complex architecture for the Home Network and a new repartition of 
the functions in the network as a whole. Some autonomic functions could be helpful to hide this complexity to the user and for keeping the management of the home network simple.

\section{References}

1. MaLigneTV. Digital TV on ADSL; http://www.malignetv.fr/

2. Very High Speed pilot program ("Fiber To The Home"); http://www.francetelecom.com/en/financials/journalists/press releases/CP_old/cp060117.ht $\mathrm{ml}$

3. GPON $2.4 \mathrm{G}$; http://www.itu.int/newsarchive/press releases/2003/04.html

4. Digital Living Network Alliance; http://www.dlna.org/home

5. Home Gateway Initiative; http://www.homegatewayinitiative.org/

6. M. Bellec, Home Broadband Home Area Network, Keynote 3, Tuesday 3rd April, European Wireless 2007

7. http://www.ieee $802.0 r g / 802$ tutorials/july06/802\%2021-IEEE-Tutorial.ppt

8. http://bwrc.eecs.berkeley.edu/Publications/2006/Presentations/Conference 42.pdf

9. J. Kephart and D. Chess, The vision of Autonomic Computing, IEEE Computer 36(1): 41-50, 2003.

10. "AUTHONE" IST Celtic Project Proposal, H. Zimmermann et al., 2007; http://www.celticinitiative.org/Projects/AUTHONE/.

11. L. Qiu, P. Bahl, A. Rao and L. Zhou. Troubleshooting wireless mesh networks. Computer Communication Review 36(5): 17-28 (2006) 